\title{
Delayed pitch comparisons and the principle of proximity
}

\author{
DIANA DEUTSCH \\ Center for Human Information Processing, University of California at San Diego \\ La jolla, California 92093
}

\begin{abstract}
Subjects compared the pitches of two tones which were separated by a retention interval during which six extra tones were interpolated. The effects were studied of varying the sizes of the melodic intervals formed by the successive tones of the interpolated sequence. It was found that error rates were lower when the interpolated sequences were composed of smaller melodic intervals; and it was argued that such sequences formed a more effective framework of pitch relationships to which the test tones could be anchored.
\end{abstract}

When pitch comparisons are made between two temporally separated tones, errors increase with a lengthening of the retention interval (Wickelgren, 1969). Further, when a sequence of extra tones is interpolated between the two to be compared, error rates vary discretely as a function of the pitch relationships between the test tones and the interpolated tones (Deutsch, 1972, 1973a, 1975a). The present study further investigates the factors influencing performance in this situation by manipulating the pitch relationships between the successive tones of the interpolated sequence. It was reasoned that in listening to such a sequence we process not only the individual tones, but also the melodic intervals between them, which then provide a framework of pitch relationships to which the test tones can be anchored. So it was hypothesized that interpolated sequences forming melodic configurations which were more easily processed would be associated with enhanced levels of performance.

There is evidence from a variety of sources that tonal sequences are processed more effectively when they consist of melodic intervals of smaller size (e.g., Attneave \& Olson, 1971; Bregman \& Campbell, 1971; Dowling, 1973; Van Noorden, 1975); and indeed the early Gestaltists applied the principle of proximity to tonal sequences in the same way as they did to visual arrays (Koffka, 1935). So it was here reasoned that interpolated sequences composed of smaller melodic intervals would be associated with higher performance levels than interpolated sequences composed of larger melodic intervals.

This work was supported by United States Public Health Service Grant MH-21001-06. Requests for reprints should be sent to Diana Deutsch, Department of Psychology, C-009, University of California at San Diego, La Jolla, California 92093.
Since previous studies had shown that error rates vary as a function of the pitch relationships between the test and interpolated tones, the first experiment compared error rates in sequences where the interpolated tones were always chosen according to the same rules, but where these tones were ordered either at random or in such a way as to minimize the average size of melodic interval. The second condition was achieved by arranging the interpolated tones in monotonically ascending or descending order.

\section{EXPERIMENT 1}

\section{Method}

Procedure. In all conditions, subjects were presented with a test tone, which was followed by a sequence of six interpolated tones and then by a second test tone. They were instructed to ignore the interpolated tones, and to judge whether the test tones were the same or different in pitch. The subjects indicated their judgments by writing " $S$ " (same) and "D" (different) on paper. The tones were $200 \mathrm{msec}$ in duration and separated by 300 -msec pauses, except that a 2 -sec pause intervened before the second test tone.

Test tones. The test cones were all drawn from an equaltempered scale (international Pitch, $A=435 \mathrm{~Hz}$ ) and ranged over an octave from Middle $\mathrm{C}$ to the $\mathrm{B}$ above. The frequencies employed (in hertz) were: $\mathrm{C}=259, \mathrm{C} \#=274, \mathrm{D}=290$, D\# $=308, E=326, F=345, F \#=366, G=388, G \#=411, A$ $=435, A \#=461$, and $B=488$. Within each condition, in half of the sequences the test tones were identical in pitch and in the other half they differed by a semitone. In half of the sequences where the test tones differed, the first test tone was higher than the second; and in the other half, the second test tone was higher than the first. All combinations of test-tone pitches were employed equally often in both conditions.

Interpolated tones. In both conditions, the interpolated tones were drawn from the same set as the test tones and were chosen at random from this set, except that no interpolated sequence contained repeated tones or tones that were identical in pitch to either of the test tones.

Conditions. There were two conditions in the experiment. In Condition 1 the interpolated tones were ordered at random, with the restriction that no more than three successive tones followed 
a unidirectional pitch change. In Condition 2, the interpolated tones were ordered monotonically; in half of the sequences, this order was monotonically ascending, and in the other half, it was monotonically descending.

There were 48 sequences in each condition, making 96 sequences in all. The sequences were arranged in random order, with no separation by condition. They were presented in groups of 12 , with 10-sec pauses between sequences within a group and 2-min pauses between groups. Subjects listened to the entire set of sequences on two separate occasions, and the results were averaged.

Apparatus. Tones were produced as sine waves by a Wavetek oscillator controlled by a PDP-8 computer, and were recorded on tape. The tape was played to subjects on a high-quality tape recorder through loudspeakers.

Subjects. Fourteen undergraduates at the University of California at San Diego served as subjects for the experiment, and were paid for their services. The subjects were selected on the basis of obtaining a score of at least $80 \%$ correct on a short tape designed as in Condition 1 (interpolated tones ordered at random).

\section{Results and Discussion}

The results of the experiment are shown in Table 1. It can be seen that error rates were indeed lower where the interpolated tones were ordered monotonically than where they were ordered at random. This effect was found to be highly significant (Conditions 1 vs. $2, \mathrm{p}<.005$, one-tailed, on a Wilcoxon test). No differences were found, within Condition 2, between sequences which were arranged in ascending order compared with descending order.

The results of this experiment are in accordance with the proposed hypothesis, that interpolated sequences forming melodic intervals of smaller size would be associated with enhanced levels of performance. However, an alternative explanation should also be considered. This is that the superior performance in Condition 2 was due, not to the reduced size of melodic interval, but rather to the fact that the interpolated sequences followed a unidirectional pitch change. There is evidence that such sequences may be more effectively processed than those where pitch changes reverse direction (Divenyi \& Hirsh, 1975; Van Noorden, 1975); and this would also be expected from the Gestalt principle of good continuation. A second experiment was therefore performed to dissociate the effect of interval size from unidirectionality of pitch change. In this second experiment, the pitch range of the interpolated tones was varied, so that comparison could be made between monotonic and random sequences where the melodic intervals were closely similar in size. If the results obtained in Experiment 1 were due simply to interval size, then no differences would be expected from such sequences, depending on whether they were ordered randomly or monotonically. However, if unidirectionality of pitch change were responsible for the reduction in errors, then monotonic sequences should result in superior performance compared with random sequences, given closely similar interval sizes. And if both factors were operating
Table 1

Percent Errors in the Different Conditions of Experiment 1

\begin{tabular}{cc} 
Condition & $\begin{array}{c}\text { Percent } \\
\text { Error }\end{array}$ \\
\hline 1 Interpolated tones ordered at random & 13.7 \\
2 Interpolated tones ordered monotonically & 8.0 \\
\hline
\end{tabular}

together, then monotonic sequences should again be associated with superior performance compared with random sequences.

There was a further difference in the design of the second experiment. Previous research had shown that interpolated sequences containing tones which were a semitone removed from the first test tone produce more errors than interpolated sequences not containing such tones (Deutsch, 1973a, 1975a). Since this effect represents a source of variance, tones which were a semitone removed from the first test tone were now excluded from the interpolated sequences. Further, since this effect has been shown to generalize from one octave to another (Deutsch, 1973b), tones were also excluded which were a semi. tone removed from the first test tone but further displaced by an octave.

\section{EXPERIMENT 2}

\section{Method}

Procedure. The procedure was identical to that in Experiment 1.

Conditions. There were four conditions in this experiment. In Conditions 1 and 2, the interpolated tones were chosen from the same set as in Experiment 1, and so ranged over an octave between $\mathrm{C}=259 \mathrm{~Hz}$ and $\mathrm{B}=488 \mathrm{~Hz}$. They were chosen at random from this set with the following restrictions: No sequence contained repeated tones and no interpolated sequence contained tones that were a semitone removed from the first test tone. In Condition 1, the interpolated tones were ordered at random, with the restriction that no more than three successive tones followed a unidirectional pitch change. In Condition 2, the interpolated tones were arranged in monotonically ascending or descending order. The average size of melodic interval in Condition 1 was 4.6 semitones; in Condition 2, this was 2.8 semitones.

In Conditions 3 and 4 , the interpolated tones were chosen from the two-octave range between $F \#=183 \mathrm{~Hz}$ and $F=691 \mathrm{~Hz}$. They were chosen at random from this set, with the following restrictions: No sequence contained repeated tones or tones which were separated by octaves. Further, no interpolated sequence contained tones which were a semitone removed from the first test tone, or which were displaced by exactly an octave from any of such tones. In Condition 3 , the interpolated tones were ordered at random, with the restriction that no more than three successive tones followed a unidirectional pitch change. In Condition 4, the interpolated tones were arranged in monotonically ascending or descending order. The average size of melodic interval in Condition 3 was 9.4 semitones, and in Condition $4,4.9$ semitones.

There were 48 sequences in each of the four conditions. Two tapes were constructed; the first contained Conditions 1 and 2, and the second contained Conditions 3 and 4 . The tapes were played to each subject twice on two separate occasions, making four listening sessions per subject in all. For half of the subjects, the order of presentation of the tapes was 1212, and for the other half it was 2121 . 
Within each tape, the sequences were arranged in random order, with no separation by condition. They were presented in groups of 12 , with 10 -sec pauses between sequences within a group and 2-min pauses between groups.

Test tones. In all conditions, the test tones were identical to those in Experiment 1.

Interpolated tones. These were drawn from the following frequencies (in hertz): $F \#=183, G=194, G \#=205, A=218$, $A \#=230, B=244, C=259, C \#=274, D=290, D \#=308$, $E=326, F=345, F \#=366, G=388, G \#=411, A=435$, $A \#=461, B=488, C=517, C \#=548, D=581, D \#=615$, $E=652$, and $F=691$. The interpolated tones in the different conditions were chosen from this set according to the rules outlined in the Conditions section.

Apparatus. The apparatus was the same as in Experiment 1. Subjects. Twenty-two undergraduates at the University of California at San Diego served as subjects for the experiment, and were paid for their services. The subjects were selected on the same criterion as for Experiment 1.

\section{Results and Discussion}

The error rates in the different conditions of the experiment are shown in Figure 1. It can be seen that monotonic ordering of the tones in the interpolated sequence resulted in enhanced performance, both when these tones were drawn from a one-octave range (Conditions 1 vs. 2) and also when they were drawn from a two-octave range (Conditions 3 vs. 4). This effect was highly significant for both comparisons ( $p<.005$, one-tailed, on Wilcoxon tests in both cases). However, it can also be seen that the error rate in Condition 1 closely matched that in Condition 4 , although in the former case the interpolated sequences were ordered at random and in the latter case they were ordered monotonically; and the average interval sizes in the two conditions were closely similar. This argues strongly against the hypothesis that unidirectionality of pitch change was influencing performance; rather, the error rates in all the experimental conditions can be well explained on the basis of interval size alone.

One might here argue that the enhanced error rates in Conditions 3 and 4 could have been based in part on the increased overall pitch distance between the test tones and the interpolated tones. However, the results of a previous experiment by Deutsch (1974) makes this interpretation most unlikely. Here subjects made pitch comparison judgments with interpolated eight-tone sequences drawn from different pitch ranges. In one condition, all the interpolated tones were drawn from the same octave as the test tones; in another condition, they were all drawn from either the octave above or the octave below the test tones; and in yet another condition, half of the interpolated tones were drawn from the octave above and the other half from the octave below, the order of octave placement being random. It was found that when the interpolated tones were all drawn from a single adjacent octave, the error rate was significant-

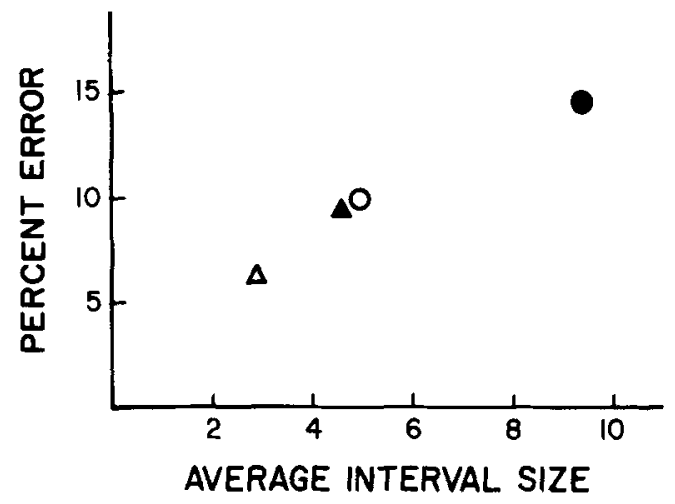

Figure 1. Percent errors in the different conditions of Experiment 2 . Interpolated tones span a one-octave range: $\Delta$ Condition 1, ordered at random; $\Delta$ Condition 2, ordered monotonically. Interpolated tones span a two-octave range: Condition 3, ordered at random; $O$ Condition 4 , ordered monotonically.

ly lower than when they were all drawn from the same octave as the test tones. Yet, on the hypothesis that a greater overall pitch distance between the test and interpolated tones produces an increase in errors, the opposite result should have been obtained. It was also found that when the interpolated tones were drawn from both the octave above and the octave below the test tones, the error rate was significantly higher than when they were all drawn from the same octave, and this is as expected on the hypothesis that an increased error rate would be associated with a larger average interval size.

In an interesting study using several different paradigms, Olson and Hanson (1977) found that under some circumstances increased errors were associated with an increased distance between the test and interpolated tones. However, in this experiment, such an increased distance was associated with a proportionately large increase in average interval size (since there were only three interpolated tones); and, indeed, Olson and Hanson suggest an interpretation of their results based on an encoding of tonal relationships which is very similar to the present hypothesis.

As in Experiment 1, no significant differences emerged within the conditions where the interpolated tones were ordered monotonically, depending upon whether this ordering was in an ascending or descending direction. It will also be noted that the error rates in Conditions 1 and 2 (interpolated tones chosen from a one-octave range) were lower than those in Experiment 1. This is as expected, since tones which were a semitone removed from the first test tone were excluded from the interpolated sequences in Experiment 2; and such tones have been shown to produce an increase in error rate (Deutsch, 1973a, 1975a). 


\section{GENERAL DISCUSSION}

The results of these two experiments are consistent with the proposed hypothesis that interpolated sequences composed of melodic intervals of smaller size are more accurately and easily processed, and so provide a more effective framework of pitch relationships to which the test tones can be anchored. Support for this hypothesis comes from a variety of sources. The most direct evidence is provided by Attneave and Olson (1971), who required subjects to transpose tonal patterns and found that, in musically naive listeners, variability in the size of reproduced melodic intervals was proportional to the size of these intervals. Further evidence for a more effective processing of smaller melodic intervals comes from studies involving the presentation of rapid sequences of tones. Bregman and Campbell (1971) found that, with sequences presented at a rate of $10 / \mathrm{sec}$, listeners had considerable difficulty in perceiving the order of succsssive tones when these were spaced far apart in pitch; however, this problem did not arise when the tones spanned a narrow pitch range. Van Noorden (1975) also found that, at fast presentation rates, tonal sequences appeared more "coherent" when successive tones formed smaller melodic intervals than when they formed larger ones. In another study, Dowling (1973) presented the notes of two wellknown melodies alternately at a rate of $8 / \mathrm{sec}$. He found that recognition of the two melodies was easy when they were in different pitch ranges; however, recognition was very difficult when their pitch ranges overlapped. Dowling attributed this finding to the more ready processing of smaller melodic intervals, which would lead to the perceptual integration of the alternating melodies in the overlapping, but not the nonoverlapping, condition. In a further experiment involving dichotic listening, Deutsch (1975b) presented subjects with a major scale, played simultaneously in both ascending and descending forms, and switching from ear to ear such that when a note from the ascending scale was in one ear, a note from the descending scale was in the other ear, and vice versa. This scale was played repetitively 10 times without pause. Most subjects reported hearing two melodies which were separated by pitch range; and here again it was argued that this percept was determined by the principle of proximity.

More general evidence is provided by the statistical distribution of melodic interval sizes in the music of various cultures. As pointed out by Dowling (1967), there is a striking tendency for the frequency of occurrence of a melodic interval to be inversely correlated with its size. This has been demonstrated in the music of various primitive cultures (Merriam, 1964),
Western cultivated music (Fucks, 1962; Ortmann, 1926), and recently in currently popular music (Jeffries, 1974). It seems plausible to suppose that such a distribution is based on an increasing inaccuracy or difficulty in the processing of melodic intervals as they increase in size. The present findings are in accordance with this line of reasoning.

\section{REFERENCES}

Attreave, F.. \& Olson, R. K. Pitch as a medium: A new approach to psychophysical scaling. American Journal of Psychology, 1971, 84, 147-165.

Bregman, A. S.. \& Campbell, J. Primary auditory stream segregation and perception of order in rapid sequence of tones. Journal of Experimental Psychology, 1971, 89, 244-249.

DeUTsCB. D. Mapping of interactions in the pitch memory store. Science, 1972, 175, 1020-1022.

DEUTSCH, D. Interference in memory between tones adjacent in the musical scale. Journal of Experimental Psychology, 1973. 100. 228-231. (a)

DEUTSCH. D. Octave generalization of specific interference effects in memory for tonal pitch. Perception \& Psychophysics, 1973, 13, 271-275. (b)

DEUTSCH, D. Generality of interference by tonal stimuli in recognition memory for pitch. Quarterly Journal of Experimental Psychology, 1974. 26, 229-234.

DEUTSCH, D. The organization of short-term memory for a single acoustic attribute. In D. Deutsch \& J. A. Deutsch (Eds.), Short term memory. New York: Academic Press, 1975. Pp. 107-151. (a)

Deutsch. D. Two-channel listening to musical scales. Journal of the Acoustical Society of A merica, 1975. 57. 1156-1160. (b)

Diveny. P. L.. \& Hirsh. I. J. The effect of blanking on the identification of temporal order in three-tone sequences. Perception \& Psychophysics, 1975, 17, 246-252.

DoWLING. W. J. Rhythmic fission and the perceptual organization of tone sequences. Unpublished doctoral dissertation. Harvard University, 1967.

Dowling, W. J. The perception of interleaved melodies. Cognitive Psychology, 1973, 5, 322-337. (b)

Fucks. W. Mathematical analysis of the formal structure of music. Institute of Radio Engineers Transactions on Information Theon, 1962, 8. 225-228.

JEFFRIES, T. B. Relationship of interval frequency count to ratings of melodic intervals. Journal of Experimental Psychology, 1974, 102. 903-905.

KOFFKA, K. Principles of Gestalt psychology. New York: Harcourt, Brace and World. 1935.

MerRIAM, A. P. The anthropology of music. Evanston: Northwestern University Press, 1964.

Olson, R. K.. \& Hanson, V. Interference effects in tone memory. Memory \& Cognition. 1977, 5, 32-40.

Ortmann. O. On the melodic relativity of tones. Psychological Monographs, 1926. 35. Whole No. 162.

VAN Noorden, L. P. A. S. Temporal coherence in the perception of tone sequences. Unpublished doctoral thesis, Technische Hogeschool, Eindhoven, Holland, 1975.

Wickelgren. W. A. Associative strength theory of recognition memory for pitch. Journal of Mathematical Psychology, 1969, 6. 13-61.

(Received for publication July 20. 1977; accepted October 1, 1977.) 